\title{
EFFECT OF A SHORT ISCHAEMIC PRECONDITIONING PROTOCOL ON 100-M FRONT CRAWL PERFORMANCE
}

original paper

(1) University School of Physical Education in Wroclaw

DOI: https://doi.org/10.5114/hm.2021.100326

\section{VICTOR SABINO DE QUEIROS ${ }^{1}$, MATHEUS DANTAS ${ }^{1}$, RÔMULO VASCONCELOS TEIXEIRA ${ }^{1}$, VICTOR MANUEL MACHADO DE RIBEIRO DOS REIS ${ }^{2}$, DIHOGO GAMA DE MATOS ${ }^{3}$, LUIZ FELIPE DA SILVA ${ }^{1}$, PAULO MOREIRA SILVA DANTAS ${ }^{1}$, BRENO GUILHEME ARAÚJO TINÔCO CABRAL ${ }^{1}$}

\footnotetext{
${ }^{1}$ Department of Physical Education, Federal University of Rio Grande do Norte, Natal, Brazil

${ }^{2}$ Research Centre in Sport Science, Health Sciences and Human Development, University of Trás-os-Montes and Alto Douro, Vila Real, Portugal

${ }^{3}$ Institute of Parasitology, McGill University, Montreal, Canada
}

\begin{abstract} formance in the 100-m front crawl swimming modality. release (reperfusion). improvement in 100-m front crawl in young athletes.

\section{Introduction}

Competitive swimming encompasses 16 Olympic swimming pool events with distances ranging from 50 to $1500 \mathrm{~m}$ [1]. Small details can be of considerable significance in this type of sport, considering that the short event (i.e., $100 \mathrm{~m}$ ) results can depend on differences of a mere $0.04 \mathrm{~s}$ [2].

Therefore, sports science researchers have been looking for ways to maximize swimmers' performance in competitive situations, such as, for example, ischaemic preconditioning (IPC) protocols [3-5]. IPC can be defined as a process that exposes a given organ (e.g.,
\end{abstract}

Purpose. The aim of our study was to analyse the effect of a single-cycle ischaemic preconditioning (IPC) protocol on per-

Methods. Overall, 16 swimmers were recruited: 8 female athletes $(12.9 \pm 0.88$ years) and 8 male athletes $(13.1 \pm 0.88$ years). In a randomized crossover design, all participants performed a 100-m front crawl sprint preceded by an IPC or placebo cycle. In the IPC trial, a pneumatic cuff was attached to the proximal thigh and was inflated at a pressure equivalent to $80 \%$ of arterial occlusion and remained inflated for $5 \mathrm{~min}$ (ischaemia); in the placebo trial, the cuff remained inflated for the same amount of time, but at low external pressure levels $(20 \mathrm{~mm} \mathrm{Hg})$. The volunteers started the test $5 \mathrm{~min}$ after cuff pressure

Results. It was not possible to verify significant differences within the time (seconds) required to complete the test between the IPC and placebo interventions (75.68 \pm 7.2 and $75.75 \pm 8.1 \mathrm{~s}$, respectively; $p=0.916)$.

Conclusions. Therefore, we can conclude that the tested IPC protocol does not seem to be sufficient to provide performance

Key words: swimming, power, ischaemia-reperfusion, vascular occlusion

skeletal muscle) to brief periods, usually $5 \mathrm{~min}$, of ischaemia (i.e., reduced blood flow) and reperfusion (i.e., reoxygenation of tissue) (IR, ischaemia-reperfusion). This method aims to reduce the deleterious effects promoted by future and prolonged ischaemic harm [6]. The technique has been shown to be a potent stimulator for releasing vasodilator substances [7], as well as promoting changes in energy metabolism in animal models (ATP preservation) [8], physiological changes of interest for sports scientists [9].

From this perspective, IPC, performed through the application of pneumatic cuffs to the thigh, was initially indicated to improve maximal oxygen uptake

Correspondence address: Victor Sabino de Queiros, Department of Physical Education, Federal University of Rio Grande do Norte, University Campus, BR 101, Natal-RN, 59072-790, Brazil, e-mail: victorsabino97@ufrn.edu.br

Received: February 18, 2020

Accepted for publication: May 17, 2020

Citation: De Queiros VS, Dantas M, Teixeira RV, De Ribeiro Dos Reis VMM, De Matos DG, Da Silva LF, Dantas PMS, Cabral BGAT. Effect of a short ischaemic preconditioning protocol on 100-m front crawl performance. Hum Mov. 2021;22(3):70-76; doi: https://doi.org/10.5114/hm.2021.100326. 
by $3 \%$ and energy production by $1.6 \%$ in an incremental cyclist test [9]. Other studies followed the IPC protocol, demonstrating beneficial effects in cyclists and long distance runners $[10,11]$. In fact, IPC seems to have an ergogenic impact in aerobic modalities; however, the results are less conclusive when it comes to tests with predominant anaerobic metabolism $[4,5$, $12,13]$, reflecting the need for a greater number of investigations.

Most studies employ 3-4 cycles of IR before physical testing, totalling 30-40 min [14], although it has been shown that a threshold for the effects of IPC is reached with only $5 \mathrm{~min}$ of blood flow occlusion, regardless of the number of cycles used [15]. Furthermore, Beaven et al. [16] found an improvement in performance 24 hours after a protocol consisting of two 3 -min cycles of thigh blood flow occlusion. Therefore, short-term IPC protocols seem to result in improved human performance, being a more advantageous option for coaches and athletes [17]. In this sense, our study aimed to analyse the effect of a protocol composed of $5 \mathrm{~min}$ of vascular occlusion (VO) in the lower limb followed by $5 \mathrm{~min}$ of reperfusion on the performance in 100-m crawl sprint. We hypothesized that the tested protocol would be able to improve the performance of the time trial.

\section{Material and methods}

\section{Participants}

The sample was composed of 16 young national level swimmers, 8 boys (13.1 \pm 0.88 years; $49.8 \pm$ $10.2 \mathrm{~kg} ; 165.5 \pm 7.9 \mathrm{~cm}$; fat percentage: $18.8 \pm 5.3 \%$ ) and 8 girls $(12.9 \pm 0.88$ years; $49.8 \pm 10.2 \mathrm{~kg} ; 161.1 \pm$ $8.04 \mathrm{~cm}$; fat percentage: $22.8 \pm 6.06 \%$ ). Evidence concerning the influence of gender on ergogenic effects of
IPC is controversial [12, 18]; however, it has previously been found that men and women have distinct physiological responses (i.e., lactate concentrations) in 100-m crawl sprint [19]. Therefore, we performed the analysis of the total group and after stratification by gender. As inclusion criteria, the volunteers should have practiced the sport for a period of at least 4 years (frequency of training: more than 3 times a week), should not have used substances with the capacity to optimize sports performance (i.e., dietary supplements, drugs), should not have had recent osteomioarticular lesions, and should have had normal blood pressure values at rest (medium value: $108.7 \pm 7.5$ and $72.3 \pm 10.2 \mathrm{~mm} \mathrm{Hg}$ for systolic and diastolic blood pressure, respectively).

\section{Experimental design}

All volunteers made 3 visits to the laboratory. The first meeting was used to collect anthropometric, haemodynamic, and body composition (X-ray dual emission densitometry [Lunar ${ }^{\circledast} /$ G.E PRODIGY - LNR 41.990, United States]) variables and to apply a familiarization test. Randomly, visits 2 and 3 were devoted to placebo or IPC application (Figure 1). The tests took place on different, non-consecutive days (7-day washout) and at the same time (18:00-21:00). The volunteers were instructed to wear the same bathing suits in the experimental and control tests. The performance evaluation consisted of a 100-m crawl sprint, preceded by the experimental protocol. All tests were performed outside the competition period and the athletes had been training 5 times a week for about $120 \mathrm{~min}$. To avoid any interference with the evaluations, the participants were instructed to abstain from any vigorous activity (including training sessions) and from consuming caffeine-containing foods or drinks within 72 hours prior to each trial.

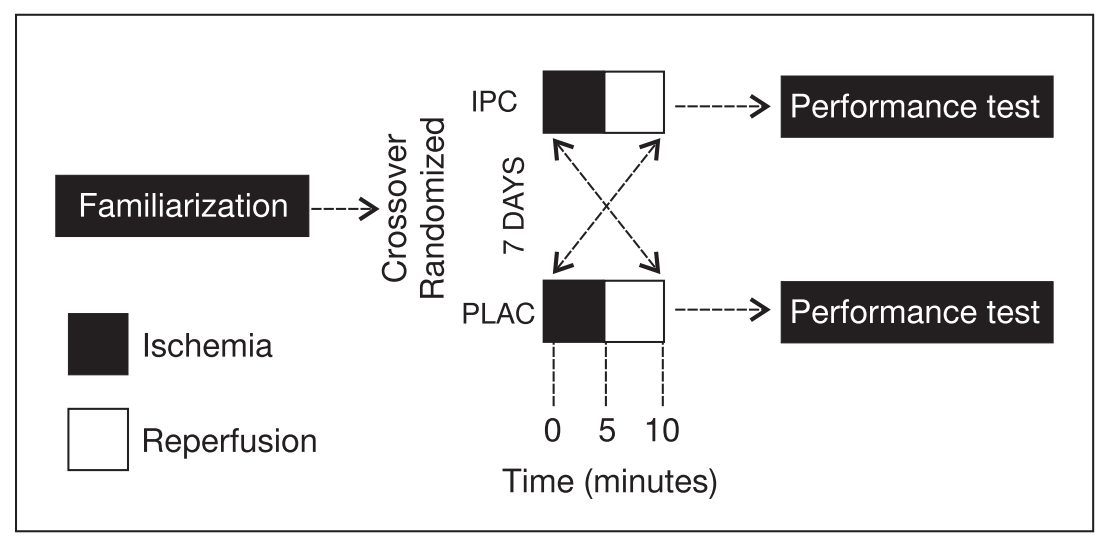

IPC - ischaemic preconditioning PLAC - placebo

Figure 1. Experimental design of the study 
Determination of blood flow restriction pressure

Total occlusion pressure (TOP) was obtained by using a highly sensitive portable vascular doppler (MedPej ${ }^{\circledR}$, DF-7001 VN, Ribeirão Preto, Brazil), after the individuals had rested for 5 min in the supine position on a stretcher. The probe was fixed above the tibial artery, with the aim to capture the auscultatory pulse. To obtain the pressure values (mm Hg) necessary to promote total vascular limb occlusion (elimination of the wrist), a standard pressure cuff was attached to the proximal part of the thigh and was inflated to the point at which the auscultatory pulse disappeared [20]. Measurements were performed on both limbs, considering that bilateral differences in the lower limb TOP levels were reported in a previous study [21], and were always initiated with the right leg.

\section{IPC and control protocol}

The IPC protocol consisted of $5 \mathrm{~min}$ of ischaemia and $5 \mathrm{~min}$ of reperfusion $(10 \mathrm{~min}=1$ cycle $)$. A pneumatic cuff $(800 \times 70 \mathrm{~mm})$ was fixed below the inguinal crease and inflated until reaching pressure values equivalent to $80 \%$ of the TOP. Previously, it was demonstrated that this relative pressure combined with lowintensity exercise induced changes in neuromuscular function [22]. The participants were asked every $1 \mathrm{~min}$ to inform the researcher whether or not they could complete the ischaemia protocol. The IPC conditions were reproduced for the placebo trial, but a protocol previously proposed was adopted in which the cuff remained inflated at $20 \mathrm{~mm} \mathrm{Hg}$ [3]. Both protocols were performed with the subjects in the supine position.

\section{Performance test (100-m crawl time)}

The volunteers entered a 25-m pool at the end of the IPC and placebo protocols and were instructed to swim $100 \mathrm{~m}$ as quickly as possible (Figure 2). Before the test, they performed dynamic stretching exercises aimed at the upper and lower limbs (2 sets - 10 repeti-

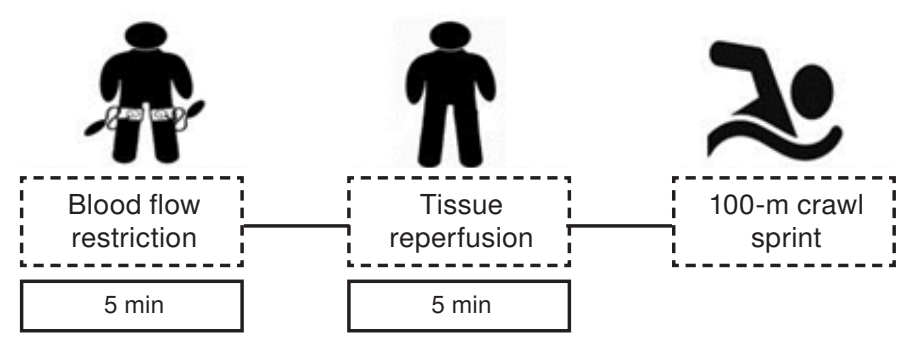

Figure 2. Outline of experimental sessions tions). The test started at the sound of a whistle, with the participants beginning from inside the pool so that their diving technique could not interfere with the results. The time required to complete the test was marked by 3 skilled and experienced sports evaluators using 3 timers, and the mean of the times was used for analysis [5]. None of the evaluators was aware of the type of intervention applied (IPC or placebo) in the participants. In addition, athletes only had access to the test time after the last collection. Immediately after the performance test, the swimmers were instructed to report their rate of perceived exertion (RPE) for that task using the Borg category-ratio 10 (CR-10) scale with visual and numerical indicators [23].

\section{Statistical methods}

Data normality was confirmed with the ShapiroWilk test. Differences between the 2 exercise conditions were investigated with paired samples $t$-tests after confirming the Gaussian distribution of data with a 5\% significance threshold. The effect sizes $(E S)$ were calculated by Cohen's $d$ (trivial ES: < 0.2; small ES: 0.2-0.6; moderate ES: 0.6-1.2; large ES: 1.2-2.0; very large ES: 2.0-4.0) [24].

\section{Ethical approval}

The research related to human use has complied with all the relevant national regulations and institutional policies, has followed the tenets of the Declaration of Helsinki, and has been approved by the Research Ethics Committee of the Federal University of Rio Grande do Norte (UFRN) (opinion: 3.464.925).

\section{Informed consent}

Informed consent has been obtained from all individuals included in this study.

\section{Results}

No significant differences were reported between the placebo and IPC conditions in the 100-m crawl performance for boys $\left(\mathrm{t}_{(7)}=-0.576 ; p=0.583 ; d=0.08\right.$; $95 \%$ CI: -3.31 to 2.01$)$, girls $\left(\mathrm{t}_{(7)}=-0.737 ; p=0.485\right.$; $d=0.11 ; 95 \% \mathrm{CI}:-1.13$ to 2.13 ), or the total group $\left(\mathrm{t}_{(15)}=0.108 ; p=0.916 ; d=0.09 ; 95 \%\right.$ CI: -1.32 to 1.46). For RPE, significant differences were identified in the total group $\left(\mathrm{t}_{(15)}=-2.406 ; p=0.02 ; d=0.58\right.$; $95 \%$ CI: -1.65 to -0.10$)$, but not after stratification by $\operatorname{sex}\left(\mathrm{t}_{(7)}=1.433 ; p=0.195 ; d=0.5 ; 95 \%\right.$ CI: -0.56 to 2.31 and $\mathrm{t}_{(7)}=1.986 ; p=0.08 ; d=1.18 ; 95 \%$ CI: -0.16 to 1.91, for girls and boys, respectively) (Figures 3 and 4). 


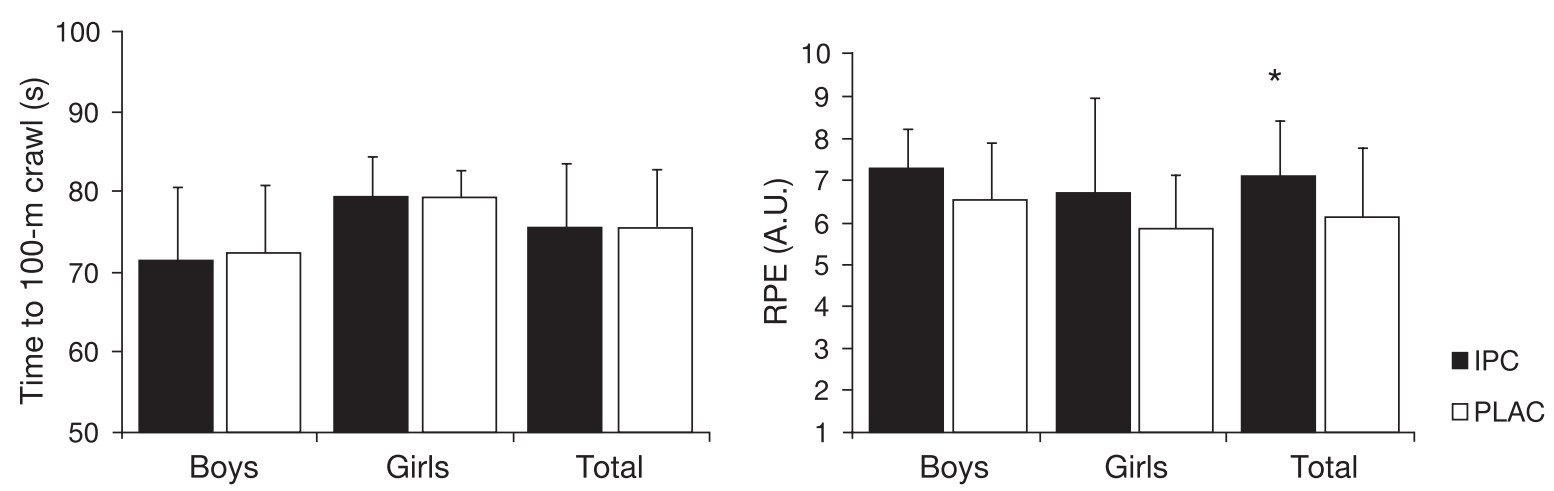

IPC - ischaemic preconditioning, PLAC - placebo, RPE - rate of perceived exertion * significant difference

Figure 3. Effect of IPC on physical performance and RPE
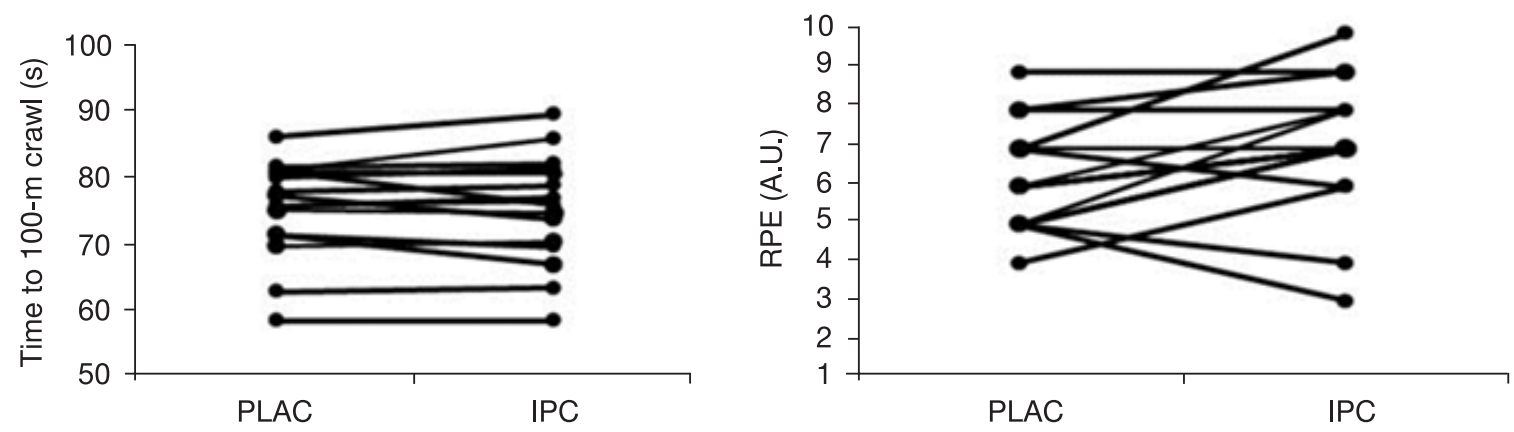

IPC - ischaemic preconditioning, PLAC - placebo, RPE - rate of perceived exertion

Figure 4. Individual responses for 100-m crawl time and RPE

\section{Discussion}

The aim of our study was to determine the effect of an IPC protocol composed of 5 min of VO, performed on the lower limb by means of a pneumatic cuff, followed by 5 min of reperfusion, on the subsequent performance in 100-m crawl. In addition, we checked the reported RPE right after the end of the time trial. It was not possible to verify differences between the interventions performed for the 100-m screening, but a higher $\mathrm{RPE}$ was reported in the IPC condition.

Previously, it was found that brief periods of ischaemia interspersed by reoxygenation of muscle tissue were able to maximize physical performance. Most often, 3-4 IR cycles are applied with a duration of 5 min, totalling 30-40 min [5, 9-11]. Some authors use this number owing to the responses observed in animal models [9]. However, Beaven et al. [16] indicated an improvement in physical performance within 24 hours after the execution of an IR protocol consisting of $2 \times 3$-min VO cycles applied unilaterally through pneumatic cuffs on the lower limbs. Therefore, shorter protocols seem to have an ergogenic effect, being a more advantageous option for technicians and athletes.
Jean-St-Michel et al. [4] found a significant improvement in 100-m swimming performance using the athlete's best stroke style after applying a 40-min upper limb IPC protocol (4 ischaemia cycles interspersed with 4 reperfusion cycles). In addition to the duration of the protocol and place of application, the study in question differs from ours in the amount of time established between the IPC and the physical tests (45 min vs. immediately after). Although there is no consensus on the ideal time for IPC to exert an ergogenic effect, some authors have reported no improvement [12, 13] and even reduced performance [25] in interventions that use short periods between IPC and anaerobic physical tests.

These findings can be justified by metabolic changes (i.e., reduction of phosphocreatine, ATP, total adenosine nucleotide content) caused by IPC in the main muscles involved in exercise [14]. When considering that the effects of IPC appear to be systemic, Salvador et al. [14] point out that IPC applied remotely may be a more advantageous option, as the athlete would benefit from the systemic effect, without the negative metabolic changes inherent in the technique. Within this perspective, we opted for the occlusion of the lower limbs, 
since they exercise less amount of work during the crawl swim [26] and proved to be capable of inducing improved performance during 50-m crawl [5]. But still, no difference between the conditions tested was observed, which may indicate the need for a longer time window between performing IPC and the physical effort of anaerobic nature.

We postulate that the time established between the IPC and the physical test also explains a higher RPE in the IPC condition. It was demonstrated that IPC applied to the lower limb (15 min of ischaemia +15 min of reperfusion) had no effect on RPE reported after water sprints; however, a minimum period of $30 \mathrm{~min}$ was established between the thigh blood flow occlusion protocol and performing the physical test [5]. The short time spent after the limb VO manoeuvre could provide an additional stimulus to exacerbate RPE, considering the theoretical model based on afferent feedback [27]. In accordance with this theory, feedback from afferents in groups III and IV (i.e., nerve endings sensitive to chemical and mechanical changes) would be the stimulus responsible for an increase in RPE [27]. Therefore, it is plausible that the accumulation of metabolic factors from the ischaemic [28] condition may have modulated RPE via afferent feedback.

Moreover, a relevant aspect of the current study is the fact that this is the first work with a sample composed exclusively of adolescent athletes, who present distinct physiology than adults [29]. For example, adolescents exhibit lower activity of certain glycolytic enzymes than their adult counterparts [29]. This characteristic should be considered, as the hypoxic condition stimulates the release of the hypoxia-induced factor 1 , an agent capable of increasing the glycolytic rate through overregulation of glycolytic metabolism enzymes [30], which can be interesting in sprint activities [31]. Therefore, the immaturity of glycolytic metabolism observed in adolescents may limit ergogenic effects in short-term activities. In addition, adolescents have lower percentages of type II fibres than adults [29], an aspect that could limit a possible post-activation potentiation effect (PAP) generated by the limb VO, as reported in another study [32]. Strengthening this theory, Arabatzi et al. [33] verified an impact of age on the effect of a PAP protocol when they found that adolescents did not benefit from a PAP protocol, but their adult peers did.

Our study has some limitations that need to be highlighted: (i) A specific heating was not used and although it has been previously suggested that heating can mitigate the effect of IPC, this procedure is present in the competitive environment. (ii) The absence of a study that would evaluate the effect of IPC in samples composed exclusively of adolescents limits our discursive capacity. (iii) We tested the application of IPC in the lower limbs; considering the hypothesis that the immediate benefits of IPC are local, the ergogenic effect of the technique could be obtained by applying the method on the arms, which perform a greater amount of work in the crawl swim.

\section{Conclusions}

The completion of 1 cycle of IPC (i.e., 5 min of VO +5 min of reperfusion) was not able to exert an immediate ergogenic effect on the performance in 100-m crawl in a sample composed of adolescents. We suggest that further studies should be performed with this population analysing the same protocol, but with a longer time between the IPC manoeuvre and physical effort.

\section{Acknowledgments}

We acknowledge the Coordenação de Aperfeiçoamento de Pessoal de Nível Superior (CAPES) for granting graduate scholarships to Victor Sabino de Queiros and Matheus Dantas.

\section{Disclosure statement}

No author has any financial interest or received any financial benefit from this research.

\section{Conflict of interest}

The authors state no conflict of interest.

\section{References}

1. Aspenes ST, Karlsen T. Exercise-training intervention studies in competitive swimming. Sports Med. 2012; 42(6):527-543; doi: 10.2165/11630760-00000000000000.

2. Hardt J, Benjanuvatra N, Blanksby B. Do footedness and strength asymmetry relate to the dominant stance in swimming track start? J Sports Sci. 2009;27(11): 1221-1227; doi: 10.1080/02640410903220336.

3. Lisbôa FD, Turnes T, Cruz RSO, Raimundo JAG, Pereira GS, Caputo F. The time dependence of the effect of ischemic preconditioning on successive sprint swimming performance. J Sci Med Sport. 2017;20(5):507511; doi: 10.1016/j.jsams.2016.09.008.

4. Jean-St-Michel E, Manlhiot C, Li J, Tropak M, Michelsen MM, Schmidt MR, et al. Remote preconditioning improves maximal performance in highly trained athletes. Med Sci Sports Exerc. 2011;43(7):1280-1286; doi: 10.1249/MSS.0b013e318206845d.

5. Ferreira TN, Sabino-Carvalho JLC, Lopes TR, Ribeiro IC, Succi JE, Da Silva AC, et al. Ischemic preconditioning and repeated sprint swimming: a placebo and 
nocebo study. Med Sci Sports Exerc. 2016;48(10):19671975; doi: 10.1249/MSS.0000000000000977.

6. Sharma V, Marsh R, Cunniffe B, Cardinale M, Yellon DM, Davidson SM. From protecting the heart to improving athletic performance - the benefits of local and remote ischaemic preconditioning. Cardiovasc Drugs Ther. 2015;29(6):573-588; doi: 10.1007/s10557-0156621-6.

7. Liu GS, Thornton J, Van Winkle DM, Stanley AW, Olsson RA, Downey JM. Protection against infarction afforded by preconditioning is mediated by $\mathrm{A} 1$ adenosine receptors in rabbit heart. Circulation. 1991;84(1): 350-356; doi: 10.1161/01.CIR.84.1.350.

8. Pang CY, Yang RZ, Zhong A, Xu N, Boyd B, Forrest CR. Acute ischaemic preconditioning protects against skeletal muscle infarction in the pig. Cardiovasc Res. 1995; 29(6):782-788; doi: 10.1016/S0008-6363(96)88613-5.

9. De Groot PCE, Thijssen DHJ, Sanchez M, Ellenkamp R, Hopman MTE. Ischemic preconditioning improves maximal performance in humans. Eur J Appl Physiol. 2010;108(1):141-146; doi: 10.1007/s00421-009-1195-2.

10. Clevidence MW, Mowery RE, Kushnick MR. The effects of ischemic preconditioning on aerobic and anaerobic variables associated with submaximal cycling performance. Eur J Appl Physiol. 2012;112(10):3649-3654; doi: 10.1007/s00421-012-2345-5.

11. Bailey TG, Jones H, Gregson W, Atkinson G, Cable NT, Thijssen DHJ. Effect of ischemic preconditioning on lactate accumulation and running performance. Med Sci Sports Exerc. 2012;44(11):2084-2089; doi: 10.1249/ MSS.0b013e318262cb17.

12. Gibson N, Mahony B, Tracey C, Fawkner S, Murray A. Effect of ischemic preconditioning on repeated sprint ability in team sport athletes. J Sports Sci. 2015;33(11): 1182-1188; doi: 10.1080/02640414.2014.988741.

13. Gibson N, White J, Neish M, Murray A. Effect of ischemic preconditioning on land-based sprinting in teamsport athletes. Int J Sports Physiol Perform. 2013;8(6): 671-676; doi: 10.1123/ijspp.8.6.671.

14. Salvador AF, De Aguiar RA, Lisbôa FD, Pereira KL, de O. Cruz RS, Caputo F. Ischemic preconditioning and exercise performance: a systematic review and metaanalysis. Int J Sports Physiol Perform. 2016;11(1):4-14; doi: 10.1123/ijspp.2015-0204.

15. Ghosh S, Standen NB, Galiñanes M. Preconditioning the human myocardium by simulated ischemia: studies on the early and delayed protection. Cardiovasc Res. 2000;45(2):339-350; doi: 10.1016/S0008-6363(99) 00353-3.

16. Beaven CM, Cook CJ, Kilduff L, Drawer S, Gill N. Intermittent lower-limb occlusion enhances recovery after strenuous exercise. Appl Physiol Nutr Metab. 2012;37(6):1132-1139; doi: 10.1139/h2012-101.

17. Marocolo M, Billaut F, da Mota GR. Ischemic preconditioning and exercise performance: an ergogenic aid for whom? Front Physiol. 2018;9:1874; doi: 10.3389/ fphys.2018.01874.
18. Paradis-Deschênes P, Joanisse DR, Billaut F. Sex-specific impact of ischemic preconditioning on tissue oxygenation and maximal concentric force. Front Physiol. 2017;7:674; doi: 10.3389/fphys.2016.00674.

19. Lampadari V, Thanopoulos V, Dopsaj M, Rozi G. Effects of age and gender in physiological responses, mechanics and performance of master swimmers. Hum Mov. 2019;20(1):17-23; doi: 10.5114/hm.2019.79393.

20. Laurentino GC, Ugrinowitsch C, Roschel H, Aoki MS, Soares AG, Neves M Jr, et al. Strength training with blood flow restriction diminishes myostatin gene expression. Med Sci Sports Exerc. 2012;44(3):406-412; doi: 10.1249/MSS.0b013e318233b4bc.

21. Neto RG, Silva JCG, Umbelino RKC, Silva HG, Neto EAP, Oliota-Ribeiro LS, et al. Are there differences in auscultatory pulse in total blood flow restriction between positions, limbs and body segments? Rev Bras Cineantropom Desempenho Hum. 2018;20(5):381-390; doi: 10.5007/1980-0037.2018v20n5p381.

22. Fatela P, Reis JF, Mendonca GV, Avela J, Mil-Homens P. Acute effects of exercise under different levels of bloodflow restriction on muscle activation and fatigue. Eur J Appl Physiol. 2016;116(5):985-995; doi: 10.1007/ s00421-016-3359-1.

23. Zamunér AR, Moreno MA, Camargo TM, Graetz JP, Rebelo ACS, Tamburús NY, et al. Assessment of subjective perceived exertion at the anaerobic threshold with the Borg CR-10 scale. J Sports Sci Med. 2011;10(1): 130-136.

24. Batterham AM, Hopkins WG. Making meaningful inferences about magnitudes. Int J Sports Physiol Perform. 2006;1(1):50-57; doi: 10.1123/ijspp.1.1.50.

25. Paixão RC, da Mota GR, Marocolo M. Acute effect of ischemic preconditioning is detrimental to anaerobic performance in cyclists. Int J Sports Med. 2014;35(11): 912-915; doi: 10.1055/s-0034-1372628.

26. Figueiredo P, Rouard A, Vilas-Boas JP, Fernandes RJ. Upper- and lower-limb muscular fatigue during the 200-m front crawl. Appl Physiol Nutr Metab. 2013;38(7): 716-724; doi: 10.1139/apnm-2012-0263.

27. Pageaux B. Perception of effort in exercise science: definition, measurement and perspectives. Eur J Sport Sci.2016;16(8):885-894;doi:10.1080/17461391.2016. 1188992.

28. Loenneke JP, Wilson GJ, Wilson JM. A mechanistic approach to blood flow occlusion. Int J Sports Med. 2010;31(1):1-4; doi: 10.1055/s-0029-1239499.

29. Armstrong N, Barker AR, McManus AM. Muscle metabolism changes with age and maturation: how do they relate to youth sport performance? Br J Sports Med. 2015;49(13):860-864; doi: 10.1136/bjsports-2014094491.

30. Papandreou I, Cairns RA, Fontana L, Lim AL, Denko NC. HIF-1 mediates adaptation to hypoxia by actively downregulating mitochondrial oxygen consumption. Cell Metab. 2006;3(3):187-197; doi: 10.1016/j.cmet. 2006.01.012. 


\section{HUMAN MOVEMENT}

V. de Queiros et al., Ischaemic preconditioning and crawl performance

31. Eynon N, Alves AJ, Meckel Y, Yamin C, Ayalon M, Sagiv M, et al. Is the interaction between HIF1A P582S and ACTN3 R577X determinant for power/sprint performance? Metabolism. 2010;59(6):861-865; doi: 10.1016/j.metabol.2009.10.003.

32. Doma K, Leicht AS, Boullosa D, Woods CT. Lunge exercises with blood-flow restriction induces post-activation potentiation and improves vertical jump performance. Eur J Appl Physiol. 2020;120(3):687-695; doi: 10.1007/s00421-020-04308-6.

33. Arabatzi F, Patikas D, Zafeiridis A, Giavroudis K, Kannas T, Gourgoulis V, et al. The post-activation potentiation effect on squat jump performance: age and sex effect. Pediatr Exerc Sci. 2014;26(2):187-194; doi: 10.1123/pes.2013-0052. 